\title{
On Framing Effects, Replications, and Scientific Inference \\ Reply to Commentary by Simmons and Nelson (2013) on Mandel (2014)
}

David R. Mandel

York University

\begin{abstract}
Author's note: A reply to Simmons and Nelson (2013) was posted at https://sites.google.com/site/themandelian/data-colada in December, 2013. As it has been cited in literature, and as the website on which it appeared will soon be inactive, the aim of this document is to ensure the reply has a permanent record going forward. This version has been edited for style, but the substantive content is the same as in the original post. Correspondence may be sent to the author at drmandel66@gmail.com.
\end{abstract}

In a recent blog post on Data Colada (hereafter, DC), entitled, "'Exactly': The Most Famous Framing Effect Is Robust To Precise Wording," Joseph Simmons and Leif Nelson (2013; hereafter S\&N; with additional input from Uri Simonsohn) report the results of a replication study of Experiment 2 in my in-press JEP: General paper entitled "Do Framing Effects Reveal Irrational Choice?" (Mandel, 2014). They contacted me a few days prior to posting to give me advanced notice and to kindly offer me the chance to give up to a 150-word reply. Since 150-words isn't enough to reply properly, they have graciously agreed to link my full reply to their blog post.

I agree with them that this is a good paper for a replication and for the reasons they give - the original work by Amos Tversky and Daniel Kahneman (Tversky \& Kahneman, 1981) is "foundational" and my critique is "novel and fundamental" (in their words). This we agree on and I welcome their attempt to replicate the findings.

There are several important issues to tackle in response to their blog post and broader issues they raise. I will start with their experiment and the conclusions they draw in their blog post and then branch out.

\section{Methods and replication quality}

The experiment S\&N replicated (Experiment 2 in Mandel, 2014) had a 2 (Frame: positive, negative) x 3 (Modifier: "at least", none, "exactly") factorial design. I used a framing problem similar to the Asian Disease Problem (ADP), which I had also used in earlier research (Mandel, 2001). S\&N stated they focused on that experiment because it was "the study most faithful to the original," meaning the Asian Disease Problem (ADP). However, it was in Experiment 1 that I actually used the ADP in a within-subjects 
design. I don't think this matters much, especially for comparing my results to S\&N's, but again, it is worth noting that I did report findings on the actual ADP.

The first step I took was to review their materials and my own. I wanted to make sure that the terms "exactly" and "at least" weren't marked in my study. The methods in my paper don't indicate that they were, but it was worth double-checking. They weren't. So, that rules out one possible source of divergence - unreported marking of linguistic modifiers. I've reviewed their materials and I agree with them that it is a precise methodological replication, except, of course, for sampling procedures.

Aside from their larger samples (a good thing), S\&N used mTurk, while subjects in my experiment were from university samples. I am not sure whether this had an effect. I think it is worth noting, and it represents an uncertainty, but I will leave it at that. I am assuming that $\mathrm{S} \& \mathrm{~N}$ use some sort of screening procedure to ensure that the subjects are fluent in English. It would be good to know what the inclusion/exclusion criteria were. And of course if they didn't, that would be important to know too.

As well, they asked subjects to recall how many people had their lives at stake, and they filtered out the roughly 50 cases that gave a wrong answer. I ran my initial tests on the comparability of our results with and without the filtering of these cases and it made little difference. I didn't use a similar filtering procedure (nor did Tversky and Kahneman), so it seems appropriate to include those cases.

\section{Results}

S\&N present the results for the "no modifier" and "exactly" conditions on their blog post and link the full set of results with the "at least" condition for readers wanting to see the full set. They reported the results in the two formats I provided: percentages of subjects choosing the certain option and their choices weighted by strength of preference. I reported the percentages mainly because most earlier papers don't use the more sensitive weighting method, but it makes sense to do so because it seems worth knowing whether choosing a given option was the result of a strong preference for that option or little more that a coin toss (or somewhere in between).

In the analyses that follow, S\&N and I have coded the choice of the certain option as 1 and the choice of the uncertain option as -1 and multiplied those by their preference rating $(0=$ equally preferable; $10=$ much more preferable), so weighted choice can range from -10 (strongest preference for the uncertain option) to 10 (strongest preference for the certain option), with 0 representing indifference.

Our analyses concur, and I'll present their filtered results for simplicity, although I'll later run tests on both the filtered and unfiltered samples. 
Here are the percentages choosing the certain option in all conditions in both studies:

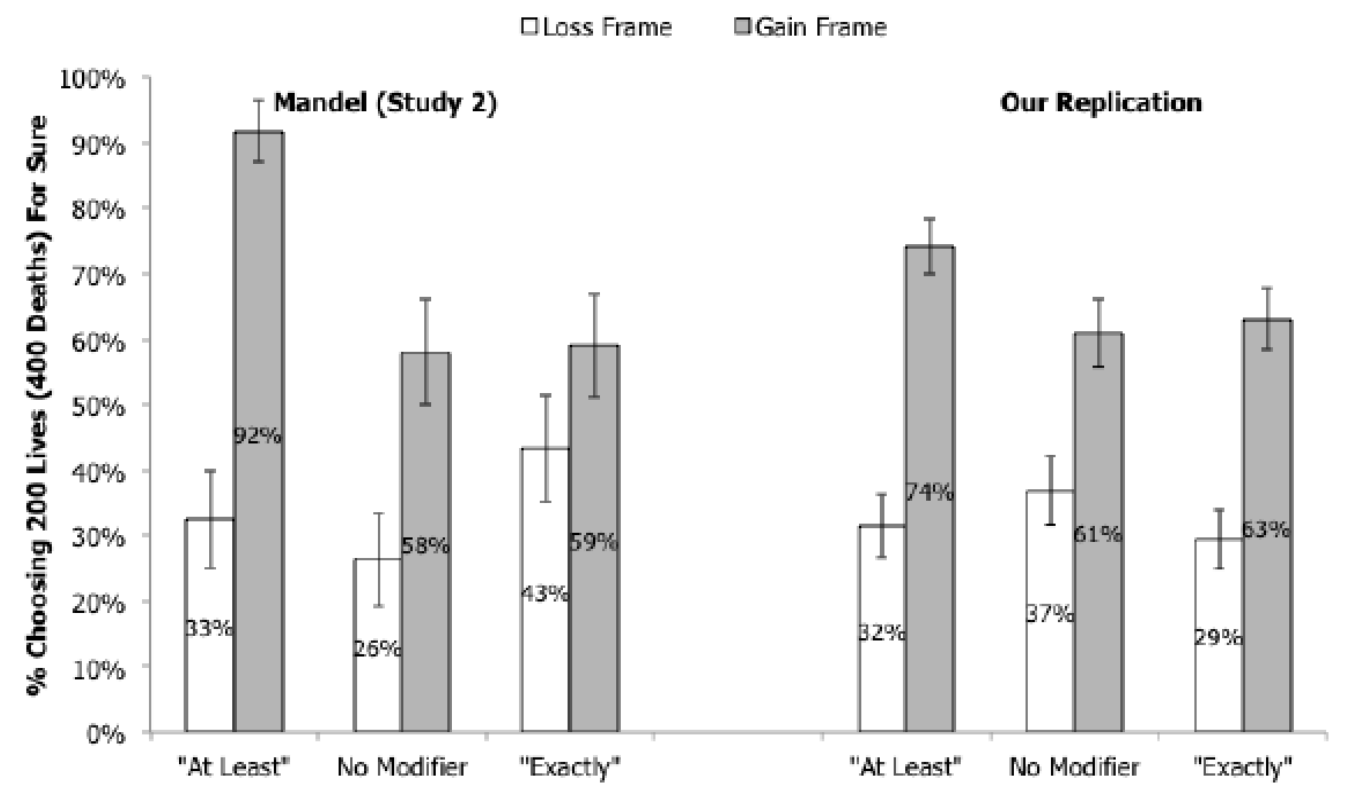

And here are the mean weighted choice findings:

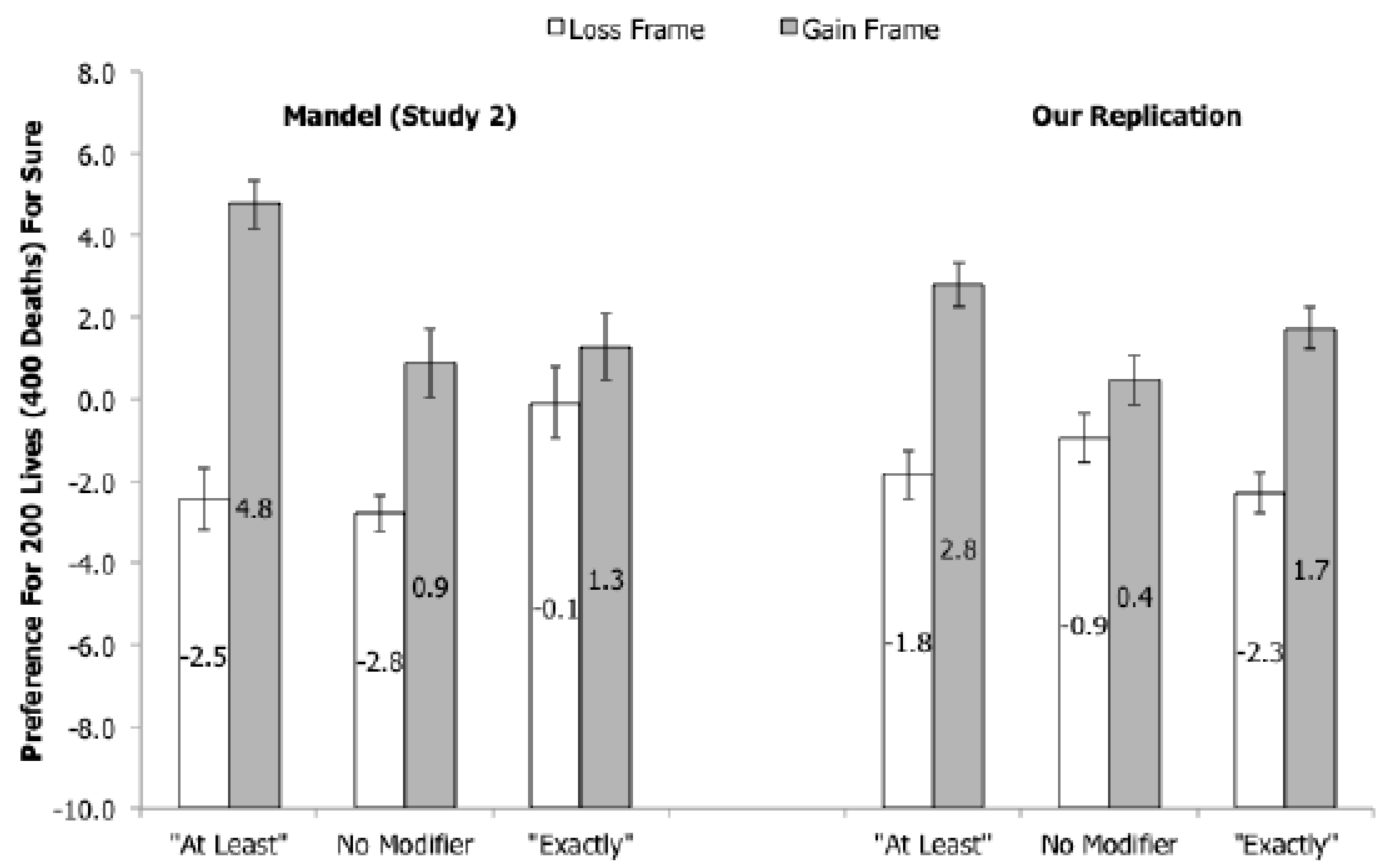




\section{S\&N's conclusions}

S\&N reported that "Unlike Mandel, we found a strong framing effect [in the unweighted choice data] even with the use of the word "exactly" $(p<.001)$." Likewise, they reported that the weighted choice data "also failed to replicate his result."

On the basis of this, $\mathrm{S} \& \mathrm{~N}$ conclude:

"In sum, our replication suggests that Tversky and Kahneman's (1981) framing effect is not caused by this linguistic artifact."

They close the post by noting that, in then end, none of this was necessary since their colleague, Uri, runs the ADP in class every year with his students using exactly and he still gets the framing effect.

Case closed.

\section{Response}

Pure replication studies are important. I think we all agree that they add important information when done properly and when appropriate conclusions are drawn. I've already noted that I find this to be a pure replication, except for sampling variations, which may or may not account for any differences. I am not claiming that they do, nor denying that they might.

However, I don't think S\&N's conclusions about my study's replicability, nor their conclusions about the theoretical significance of the results, follow quite as clearly from their findings as they suggest.

\section{The replication}

Let's begin with replicability since that is the proximate issue. Let's also be explicit about what replicability refers to here: Experiment 2 from my studies. I reported three studies (four, if you count the demonstration experiment I reported in the General Discussion, which used the limited-vaccine version of the ADP adapted by Jou, Shanteau, \& Harris, 1996). Each of those studies had a different design and tackled the issues in different ways. I have always regarded Experiment 2 as the weakest of the bunch, but I'll come back to that.

There were six experimental conditions in Experiment 2. Let's first take a look at the unweighted choice data. Here are the framing effects per condition in the original and replication experiments. Negative values indicate standard framing effects, i.e., more subjects choosing the certain option in the positive (gain) frame than in the negative 
(loss) frame. The first figure shows the results with S\&N's filtering procedure and the second without filtering.

The error bars are 95\% confidence intervals generated using Geoff Cummings' $\mathrm{ESCl}$ program for Mac, which I've used for much of the analysis that follows.

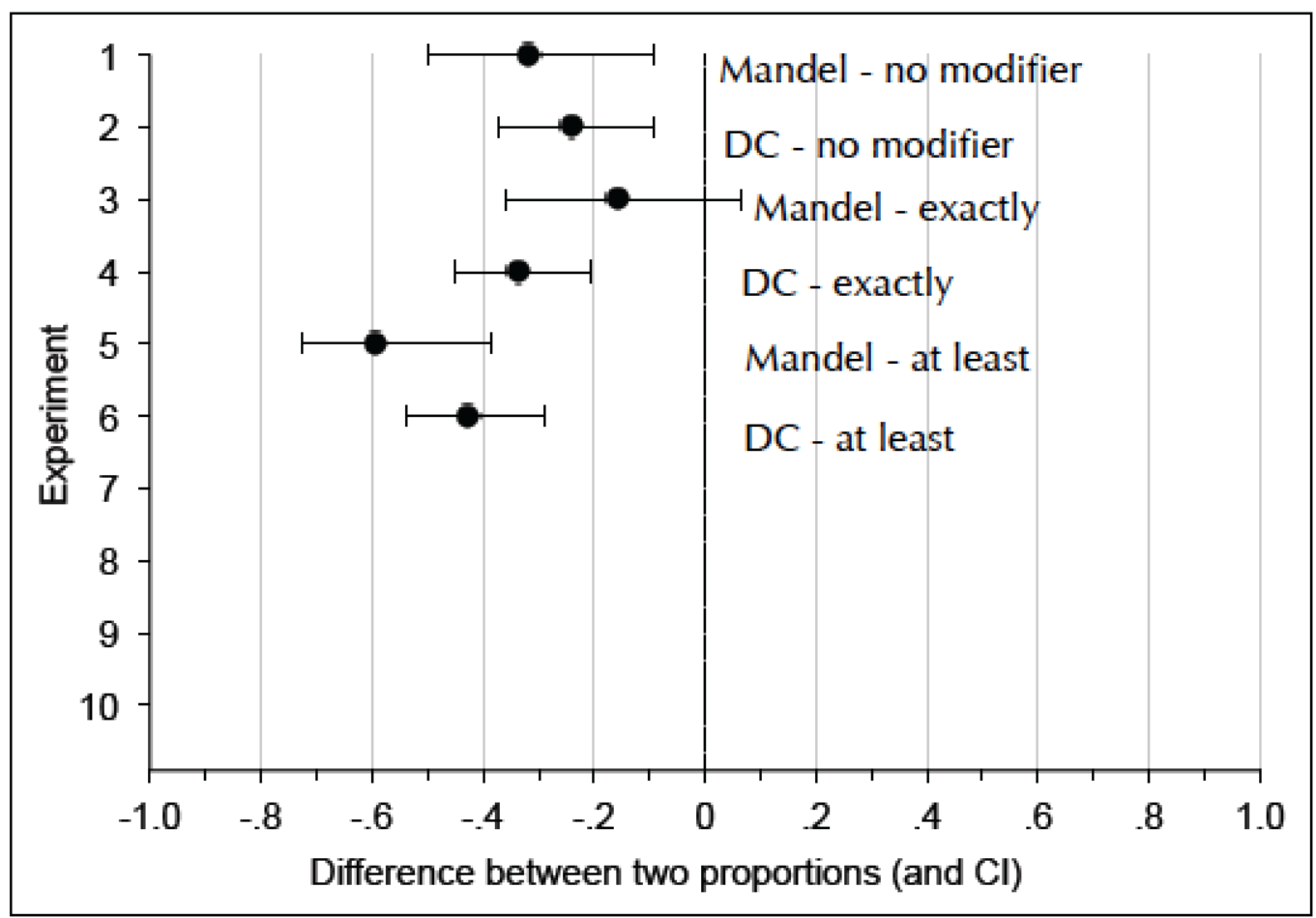

Framing effects with DC-filtered sample 


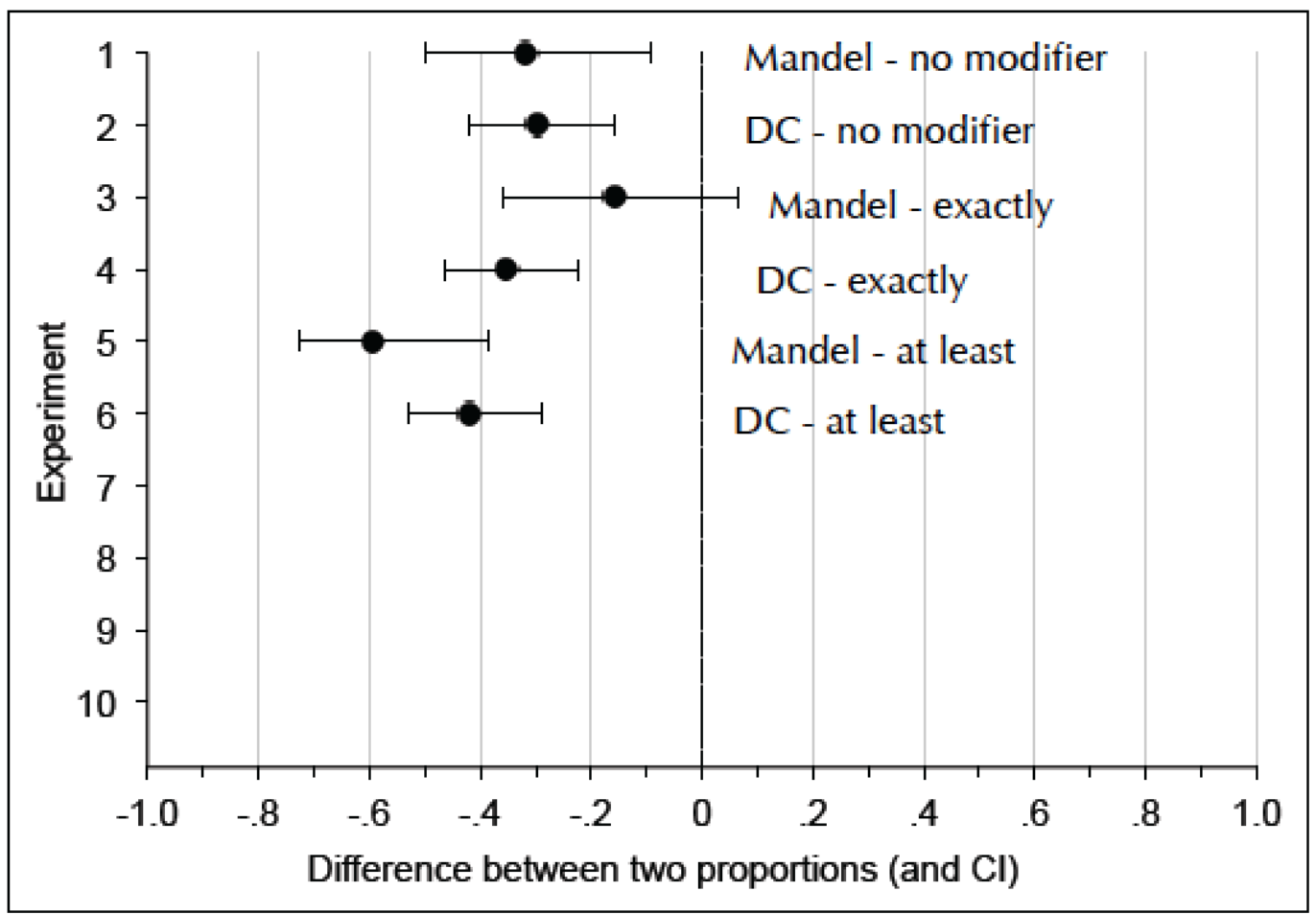

Framing effects with DC-full sample

These figures show the effects. Nothing new here, but I find it useful to see all the 95\% Cls, which show, for instance, that although my "exactly" condition was not significant, the effect was still in the direction of a standard framing effect. Also, it's evident that filtering didn't change much, so I'll move on with further analysis based on the full S\&N dataset.

The next figure compares each of the six conditions across the two experiments. 


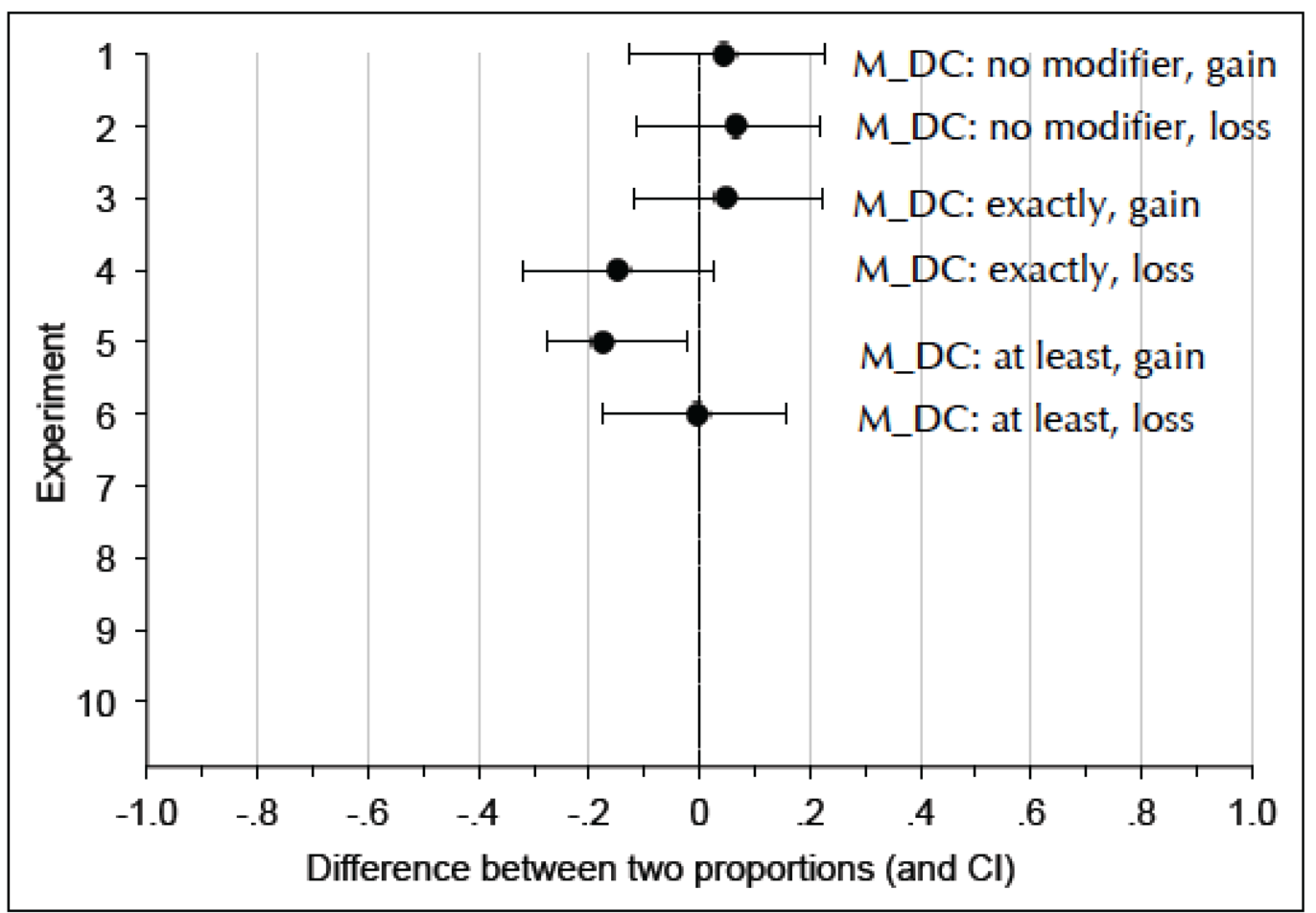

\section{Condition differences with DC-full sample}

Five out of six conditions yielded percentages of certain option choosers that did not significantly differ. There was a significant difference between the percentages in the "at least" gain frame condition, where I found a higher proportion of sure-option choosers. However, in both my experiment and S\&N's replication, that condition yielded the largest framing effect and, as one can see, the "at least" loss frame condition was virtually identical.

These six comparisons are uncorrected, and in no way theory driven. A replication study is just that - about statistical replicability of prior results. Obviously, replications that test many comparisons to the original study have a greater chance of yielding some evidence of non-replicability than those that have few comparisons. If we Bonferroni corrected, so that the overall Type I error rate was .05, then we'd need to use $99.12 \%$ Cls; i.e., $(1-.05 / 6) \times 100$. Here are the comparisons with $99 \% \mathrm{Cls}$ (close enough): 


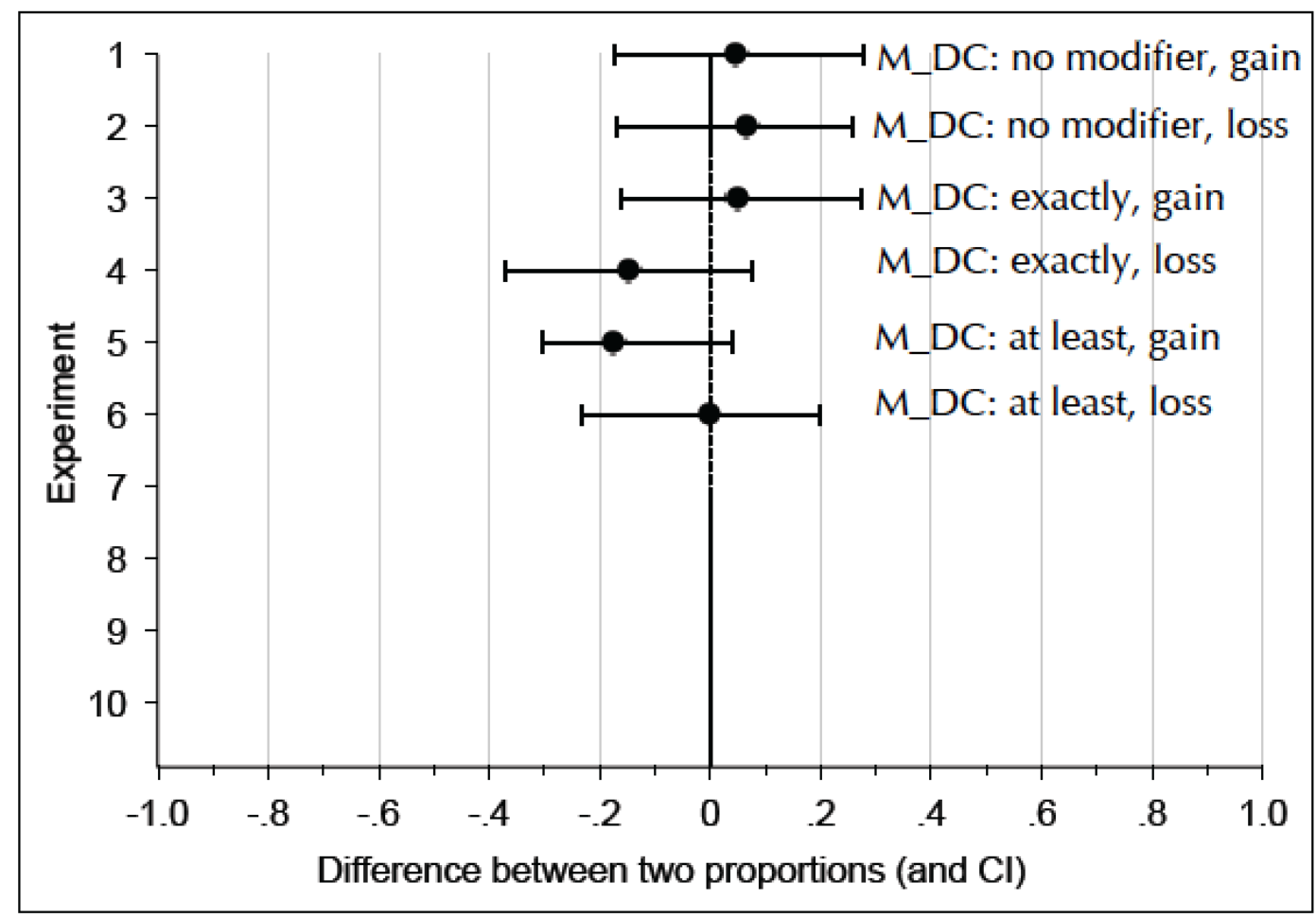

With correction for alpha inflation, none of the six conditions differed significantly between my study and S\&N's replication.

Let me say that again: using the standard metric for framing studies (percentage of subjects choosing the certain option), there was no statistically significant difference between any given condition in my Experiment 2 and S\&N's replication experiment. In other words, each condition result was replicated by S\&N.

Now that doesn't mean that the significance or nonsignificance of every reported effect will be preserved. S\&N focus on the non-replicability of my nonsignificant framing effect in the "exactly" condition. But, as we saw earlier, that effect in my study was not zero. It was in the direction of a standard framing effect. With $t(74)=1.37$, the probability of a same sign effect (prep) is $83.2 \%$ (I use Bruno Lecoutre's LePrep to generate prep and psrep). S\&N got that, and a heck of a lot more. The probability of a significant same sign effect (psrep) at a two-tailed probability of 0.00000005 (which is the exact twotailed probability corresponding to their $\mathrm{t}$ value of 5.64 with $\mathrm{df}=225$ yields) is $9.03 \mathrm{E}-04$. So their result is indeed unexpected, given my result. Note, however, that a significant effect at alpha $=.05$, given my effect, is not that surprising: the psrep $=0.283-\mathrm{a} 28.3 \%$ chance of a significant replication.

Note, however, that S\&N's "exactly" framing effect is not all that probable even given their own "no modifier" framing effect obtained from the same study using the same subject base. The psrep $=.17$. That is, we would expect not to achieve their "exactly" 
significance level $83 \%$ of the time based on their "no modifier" result. Indeed, their effect in the critical "exactly" condition is very much like their "at least" condition. Given their "at least" framing effect, with $\mathrm{t}(213)=6.77$, psrep at a two-tailed probability of 0.00000005 is .78 .

So, what S\&N's results seem to bizarrely suggest is that subjects interpret the term "exactly" to mean "at least" (linguists, please keep calm!), or perhaps that explicit modification of quantifiers somehow (how?) affects framing effects in a similar way, amplifying them relative to no explicit modifier on the numeric quantifiers. These interpretations are more consistent with their findings than the explanation that the unmodified quantifiers are treated as meaning "exactly N lives saved/lost."

S\&N don't attempt to explain why their "exactly" result is so strong and what that implies given the strength of their other two framing effects. Rather, they contrast my null (not nil!) effect with their very strong effect. Even without their replication, we knew there's close to a 3/10 chance of getting a significant effect at .05 given my null effect. So, a significant effect at that level in a replication isn't highly improbable. (To know this, they didn't even have to ask Uri.) The improbability of S\&N's result, however, must be interpreted not only in light of my findings (as an improbable replication result), but also in terms of their own findings. I personally cannot make sense of why explicitly modifying the quantifiers in the certain option with "exactly" would strengthen the effect as much as "at least" did. It makes no sense to suggest that "exactly" means "at least" to most people. Prospect theory doesn't predict that effect. So, while S\&N's "exactly" framing effect is an improbable result given mine, it is also a perplexing result, given their other results.

I don't want to belabour the point by repeating all of this with the weighted choice results. Let me just highlight a few observations. If one calculates Cohen's $d$ for every framing effect in the two studies, as I've done in the ESCI Meta-Analysis of $d$ Excel file here, you can test for effect heterogeneity (a significant $\mathrm{Q}$ indicates heterogeneity). What you will find is that:

(a) S\&N's "no modifier" and "exactly" conditions yield heterogeneous effects, $\mathrm{Q}=$ $4.15, p=.0418$.

(b) S\&N's "at least" and "exactly" conditions yield homogenous effects, $Q=0.01, \mathrm{p}=$ .9231 .

(c) The "no modifier" effects in Mandel and S\&N are homogenous, $Q=1.02, p=.3121$.

(d) The "exactly" effects in Mandel and S\&N are heterogeneous, $\mathrm{Q}=3.91, \mathrm{p}=.048$. 
(e) the heterogeneity in (a) and (d) is comparable. For instance, consider $\mathrm{I}^{2}$, which is a measure of the percentage of variation across studies or conditions that is due to heterogeneity rather than chance. $I^{2}=75.9 \%$ for S\&N's comparison of their "exactly" and "no modifier" weighted-choice framing effects, whereas $\mathrm{I}^{2}=74.4 \%$ for the comparison of the S\&N's and my "exactly" weighted-choice framing effects.

So much for the proximate issue.

\section{Broader issues}

Among the broader issues that S\&N's replication raises, an important one is how to make the most out of replication studies. Replications take time that could easily have been spent elsewhere. So, having conducted them, it makes sense to treat them carefully. This is not only to make the investment of time worthwhile, but, more importantly, so that they end up making a useful contribution to science.

The replication experiment may start out as a pure test of replicability, but replication researchers should be willing to triangulate the results of their findings with the body of evidence on the topic. That involves also considering the results they obtain not only in light of the original experiment's findings, but also in light of their other findings.

Ultimately, replication is merely one aspect of evidential triangulation. As scientists, we may not do enough of it, but replication researchers are not freed from the other burdens of triangulation: evaluating their findings in light of the larger body of evidence. For instance, when S\&N dismissively state, "We could have just asked Uri", my immediate thought was "Well, I could have just pointed to Karl", since Karl Teigen and colleagues have shown in various published studies that people are prone to lower bounding numerical quantifiers. Those studies also include ADP adaptations (in particular, see Teigen \& Nikolaisen, 2009). For instance, forecasters of outcomes such as "you will keep/lose $\$ 200$ " are seen to be less accurate when one ends up keeping or losing $\$ 180$ (inconsistent with "at least") than when one ends up keeping or losing $\$ 220$ (inconsistent with "at least"). This is the case for positive and negative outcomes, so outcome desirability is ruled out as an alternative explanation. Or I could have pointed to the neo-Griceans and work on scalar implicature.

The point is that even a "pure replication" ought to be contextualized. Doing that well may end up taking more time than running the replication study itself and summarizing the findings. But without that deeper commitment, replications may not serve the greater aim of evidential triangulation. It is what you do with evidence that counts.

For instance, S\&N seem to believe that their finding of a strong framing effect with "exactly" somehow reinstates prospect theory as a viable theoretical candidate for ADP-type risky choice framing effects. It doesn't. There is already strong evidence that 
ADP-style framing effects are not due to the psychophysics of valuation as captured in the value function model of prospect theory. Kühberger (1995) was the first to show that merely fleshing out the unexplicated part of the sure option within a given frame (so that the certain option matched the risky option in terms of stating explicitly that there were mixed outcomes for the sure option and a mixed set of extreme outcomes for the risky option) was sufficient to eliminate the framing effect. To be clear, if the gain frame is "If Plan A is adopted, 200 will be saved and 400 won't be saved" and the loss frame is "If Plan A is adopted, 400 will die and 200 won't die", the framing effect is eliminated. This modification actually strengthens the parallel construction of the framing manipulation across paired options, since now there are explicit mixed outcomes (i.e., positive and negative outcomes) noted in both options, and the methodological tweak in no way changes prospect theory's predictions, yet it obliterates the effect. Moreover, reversing the asymmetry actually reverses the effect, yielding framing effects that are opposed to the directional effects predicted by prospect theory. Experiment 3 in Mandel (2014) independently replicates those effects, and earlier experiments in Mandel (2001) show that framing effects are eliminated when parallel explication is achieved by truncating the risky option so that the expected utility of explicated outcomes are matched. Mike Tombu and I have studies currently under review that show similar reversals based on manipulation of explication [these studies were subsequently published in Tombu \& Mandel, 2015].

Now, one might be inclined to say that, even if those points were valid, and even if those studies showed what they did, there is still so much evidence in support of prospect theory, that it is people like me who have failed to triangulate the evidence. I mention this because I've already encountered it a number of times, not because it's a good argument. It isn't. It might be a good argument if I were claiming that prospect theory is wrong, but I don't claim that. What I actually concluded about prospect theory is "although prospect theory's value-function model has much evidence to support it from a wide range of experimental tasks (e.g., simple lotteries), it cannot accommodate the present findings." Not exactly a scathing critique. Indeed, I do not deny that prospect theory may do a good job of explaining certain types of framing effect. And, I am not "anti-framing" either, whatever that might mean. I mention it, strange as it sounds, because one reviewer seemed to think I was trying to prove that framing effects don't exist. Fortunately, I have done some work (e.g., Mandel, 2008) showing that they do exist too, which helped my case, but imagine the fun I had.

I hope that if one reads my paper, they will be left with a clear sense that the core issue I was dealing with concerned the validity of inferences made by researchers about the linguistic interpretation of quantifiers that, in turn, affect how researchers characterize the logical coherence and rationality of the "decision-makers" they study. That's why I regard Experiment 3 as the most important. Experiment 3 took a heterophenomenological approach, while Experiments 1 and 2 did not. 
That is, Experiment 3 explored how subjects interpreted the numeric quantifiers in the certain options as well the probabilities in the uncertain options. That allowed me to disaggregate the sample based on interpretational differences. When I did that, I found that subjects who said they had an "at least" interpretation of the certain option showed a standard framing effect, while those who said they had an "exactly" interpretation showed no significant effect, and a minority who said they had an "at most" interpretation showed a reversed framing effect. I have included these effects in the effect size file I linked earlier. They show stronger separability of effects than in Experiment 2, as shown here:

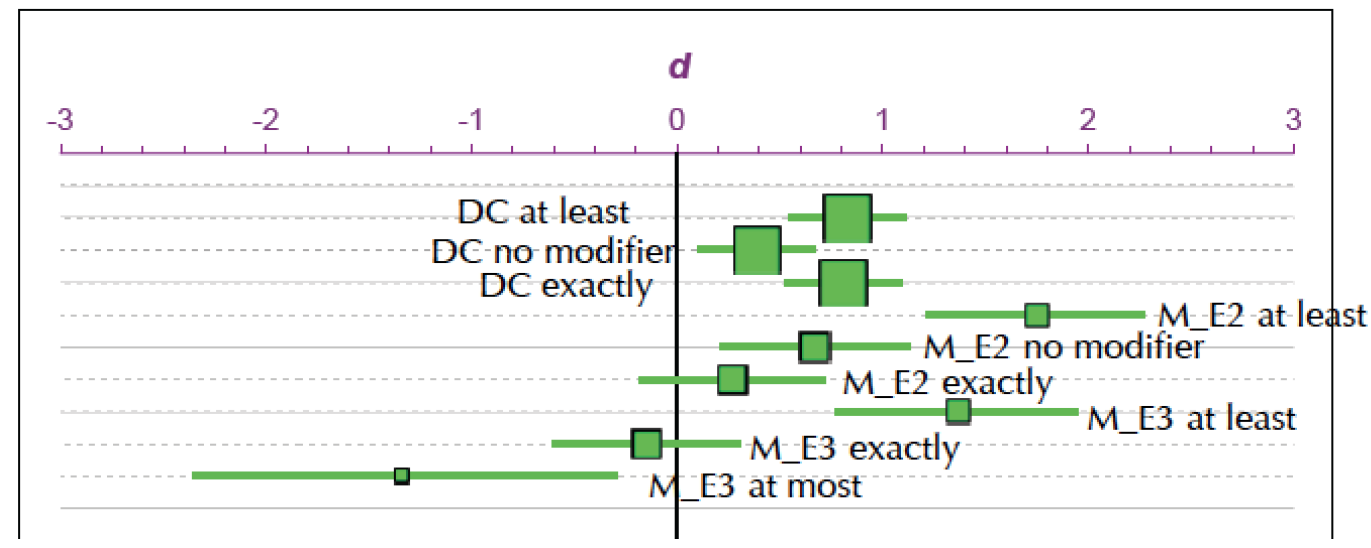

I also found that the likelihood of adopting an "at least" interpretation of the quantifier in the sure option was contingent on the extent to which that option was explicated. Subjects were more likely to adopt an "at least" interpretation when the sure option was not fully fleshed out, as in the ADP. However, even in the standard ADP-style condition, "only" 64\% adopted the "at least" interpretation, a figure that plummeted to about $24 \%$ when the sure option was fleshed out. And, in the study I reported in the General Discussion, I showed that a variation to the ADP could make "at most" a likely interpretation of the sure option in the gain frame (namely, when only 200 can be saved because there are only 200 vaccines). I note all of this because reading the DC blog post might leave readers believing that I had proposed that people invariably interpret numeric quantifiers as meaning "at least" n. In fact, I make a point of stating explicitly that I reject that view (what I've called naive unilateralism). I don't only make the point, I present evidence in the paper that does not support that view. And, Mandel and Vartanian (2011) presented evidence a few years that showed that subjects interpret the quantities in the fMRI framing task devised by DeMartino, Kumaran, Seymour, and Dolan (2006) as exact values. For those who may be unfamiliar with that study, we asked 21 subjects (DeMartino et al. had 20 subjects) in counterbalanced order: 
Imagine you are in London England and encounter the following situation:

First, you receive $£ 50$. However, you must choose one of the following options:

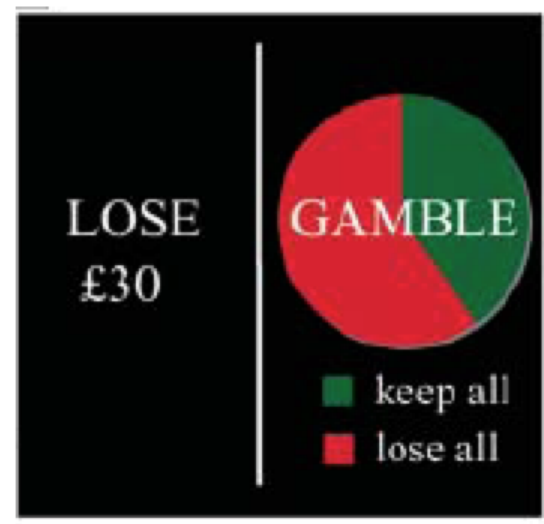

Indicate your choice by checking the appropriate box:

$\square$ Lose $£ 30$

Take the gamble

|While contemplating the statement "Lose $£ 30$ " did you consider it to mean:

$\square$ Lose at least $£ 30$.

$\square$ Lose precisely $£ 30$.

$\square$ Lose at most $£ 30$. 
Imagine you are in London England and encounter the following situation:

First, you receive $£ 50$. However, you must choose one of the following options:

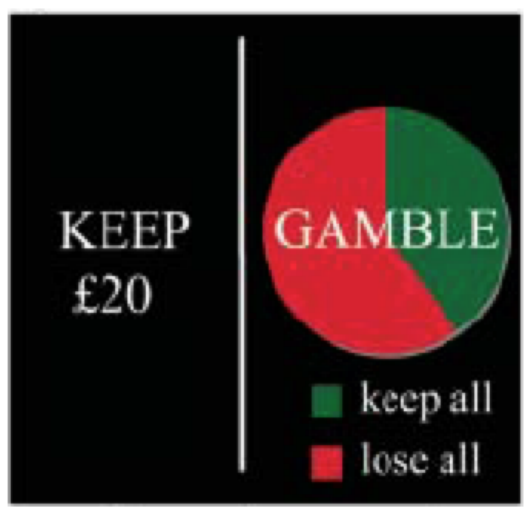

Indicate your choice by checking the appropriate box:

\section{Keep $£ 20$}

Take the gamble

While contemplating the statement "Keep $£ 20$ " did you consider it to mean:

Keep at least $£ 20$.

Keep precisely $£ 20$.

Keep at most $£ 20$.

We found that 17 out of the 21 said "precisely" in both conditions. That's $81 \%$ with $95 \% \mathrm{Cl}[60 \%, 92.3 \%]$. There was, however, no framing effect evident in our impure replication.

\section{Evolving standards for replication research, and research, more generally}

The choice of what to replicate is not value-neutral, even if a decision is made to report whatever one finds. Had S\&N regarded it as obvious that prospect theory were not a viable, if not the most viable, contender for explaining ADP-type problems, then would it have paid for them to replicate my study? Of course not. As S\&N explained at the outset of their post, they regard my studies as challenging "foundational" work (i.e., prospect theory) that they teach to their students. Likewise, had the ADP been a framing task that nobody paid attention to, would it have made sense for me to run studies showing that it might actually not be a good manipulation of strict framing? Of course not, and I say as much in the paper. 
The value-laden basis of scientific inquiry (at least in such respects) is a good thing, and there's no need to hide it. Why shouldn't we be working on what we think is important or interesting, or testing the theories we sense are better on track than others, or attempting to replicate the ones we have our doubts about? Of course we should. But, we also should take precautions, and an important one is consistency in standards of assessment.

In the present case, S\&N present a dismissive assessment of my paper on the basis of their replication experiment. Yet, little over a month ago, they published a blog post entitled "Forthcoming in the American Economic Review: A Misdiagnosed Failure-toReplicate", where they challenged the authors of a replication study that apparently claimed to have failed to replicate an anchoring effect reported by Dan Ariely and colleagues. One of their critiques is that the original paper was a multi-study paper, but the replicators only attempted to replicate one study. (Sounds familiar?) Another was that, although the effect size was smaller and the effect was nonsignificant in the replication, the two effect sizes had not been subjected to proper statistical comparisons with confidence intervals. When they did that, they found that the replicators' effect size had a $95 \% \mathrm{Cl}$ that included the original. These are all good points (buttressed in that case too by Uri's classroom data), and in many ways quite similar to the ones I've made in my own remarks on their replication study. Why do Simmons and Nelson (with Simonsohn) use such arguments when they serve to support their preferred argument, but ignore such points when they do not serve the point they wish to make? Such inconsistency does not help to advance science.

Replication researchers should clearly outline what they are attempting to replicate, and what they regard as their criteria for concluding that a study was or was not successfully replicated. We need transparent standards and consistent adherence to them if we are to make the most of science. I think such standards should specify ground rules for assessing not only comparative consistency (replication), but also internal coherence (do the findings across conditions in the replication make sense?). Rules that militate against selective focusing should resonate well with the authors, who have written about the topic more generally.

Criteria should be more transparent and consistently applied and, likewise, statistical reporting should be more precise. Simmons, Nelson, and Simonsohn (2012) make a good case for their 21 words of disclosure, arguing that behavioral scientists devote far too time and space on standards for formatting documents, having clearer directives about such things as whether to put a period after a measure unit than about how to disclose methods and decisions about statistical analyses. Likewise, I hope my preceding analysis highlights the lunacy of restricting statistical reporting to three decimal places and using imprecise $(p<.001)$ conventions. This does not aid clarity or lend itself to secondary analysis. I would rather know that S\&N got a significant framing effect in their "exactly" condition at a two-tailed $p=0.00000005$ than to learn it was 
less than .001. Surely, we can save a bit of space in our papers to accommodate the kind of statistical precision that would facilitate better evidential triangulation over time. One percent less speculation should be more than enough to meet this requirement (and leave ample room for Simmons et al.'s 21 words).

\section{Final thoughts on framing}

All of this got me thinking about, and rereading, an email exchange that Daniel Kahneman and I had over this topic back in 2010, when I first shared some of the findings from Mandel (2014) with him. We seemed to agree that an exact interpretation of the quantifiers was an unlikely reading of the ADP. He said that he and Amos had the sense that the most probable interpretation was "about 200 " or "about 400 ." I share that intuition. After all, in the ADP, we're dealing with a future epidemic. If subjects are asked their interpretation, and given the option to choose "at least", "at most", "exactly" or "about", we agreed that "about" would likely be the most common response. However, we seem to have differed in terms of the inference we would draw from that finding. I think he viewed it as showing that I left the best interpretation out of the range of options. However, I saw (and still see) "about" as an easy, noncommittal response to give, which would boost its likely selection. I predict that if "about" interpreters were pressed to resolve their uncertainty by indicating if it would be more likely that more or less than 200 were saved or that 400 would die, subjects would be more likely to indicate more, consistent with a lower-bound "at least" interpretation. In other words, the resolution of "about" would not, according to my prediction, be symmetrically distributed around 200 or 400 . For "about" not to matter in the ADP, that distribution would have to be symmetric. Otherwise, description invariance is violated and the problem ceases to be a strict framing problem.

Kahneman also noted that it was subjects' sheer sense of surprise (or of being dumbfounded) when confronted with their inconsistency of choice (in within-subject studies) that made him doubt a linguistic interpretation of the effect. That's a wonderful point. If subjects interpret the values 200 and 400 as lower bounds, then why don't they simply say so when confronted with their choices? That is, why don't they simply point out that at least 200 saved out of 600 is objectively better than at least 400 dying out 600. One explanation is that it's because they don't have lower-bound interpretations. They have either precise ("exactly") or imprecise ("about") representations of quantity that are more or less symmetric. Another possibility, however, which as I recall, Karl Teigen had first suggested to me, is that those within-subject "insight" studies seem to indicate that numeric quantifier interpretations are labile. When frames are presented separately, the quantifiers may tend to be treated as lower bounds in the ADP, but when subjects see 200 out of the 600 right next to 400 out of the 600 , that representation is cancelled and they now have an "exactly" interpretation. Their surprise would make sense if they were themselves unaware of how context had 
changed their representation, and we know from ample research in psychology that people are often unaware of what influences their thinking and behaviour.

These various ideas suggest many studies worth pursuing. I find it amazing that a topic like risky choice framing has been so heavily focused on a psychophysical account of valuation for so long. Of course, the ADP was motivated by prospect theory, and if theories were bodies, it would have the gravitational force of a star, but framing obviously also deals with mental representation, semantics, pragmatics, and how these processes affect judgment, evaluation, and decision-making. I hope we will see a broadening of our focus on this topic in the years to come.

\section{References}

De Martino, B., Kumaran, D., Seymour, B., \& Dolan, R. J. (2006). Frames, biases, and rational decision-making in the human brain. Science, $313,684-687$.

Jou, J., Shantau, J., \& Harris, R. J. (1996). An information processing view of framing effects: The role of causal schemas in decision making. Memory \& Cognition, 24, 1-15.

Kuihberger, A. (1995). The framing of decisions: A new look at old problems. Organizational Behavior and Human Decision Processes, 62, 230-240.

Mandel, D. R. (2001). Gain-loss framing and choice: Separating outcome formulations from descriptor formulations. Organizational Behavior and Human Decision Processes, $85,56-76$.

Mandel, D. R. (2008). Violations of coherence in subjective probability: A representational and assessment processes account. Cognition, 106, 130-156.

Mandel, D. R. (2014). Do framing effects reveal irrational choice? Journal of Experimental Psychology: General, 143, 1185-1198.

Mandel, D. R., \& Vartanian, O. (2011). Frames, brains, and content domains: Neural and behavioral effects of descriptive content on preferential choice. In O. Vartanian \& D. R. Mandel (Eds.), Neuroscience of decision making (pp. 45-70). New York, NY: Psychology Press.

Simmons, J. P., Nelson, L. D., \& Simonsohn, U. (2012). A 21 word solution. Available at SSRN: https://ssrn.com/abstract=2160588 .

Teigen, K. H., \& Nikolaisen, M. I. (2009). Incorrect estimates and false reports: How framing modifies truth. Thinking \& Reasoning, 15, 268-293. 
Tombu, M., \& Mandel, D. R. (2015). When does framing influence preferences, risk perceptions, and risk attitudes? The explicated valence account. Journal of Behavioral Decision Making, 28, 464-476.

Tversky, A., \& Kahneman, D. (1981, January 30). The framing of decisions and the psychology of choice. Science, 211, 453-458. 